\title{
COMPARATIVE STUDY FOR FINGERPRINT AUTOMATIC IDENTIFICATION TECHNIQUES
}

\author{
Mohamed Kamel Ahmed ${ }^{1}$; Ibrahim Ismail Ibrahim ${ }^{2}$; \\ Zaki Bassiouny Nousair ${ }^{3}$ and Noha Mohamed Ahmed ${ }^{4}$ \\ Faculty of Engineering, Helwan University, Cairo, Egypt \\ ${ }_{3}^{1} \frac{\text { mohamed._kamel@hotmail.com }}{\underline{\text { znoussair@yahoo.com }}} \quad 2 \frac{\text { iiibrahim@softhome.net }}{\underline{\text { nona_saleh@yahoo.com }}}$
}

(Received September 14, 2005 Accepted December 12, 2005)

This paper evaluates three different image representation techniques relative to fingerprint image modeling. The most popular image representation techniques such as Gabor filters, Discrete Fourier transform and Wavelet transform will be implemented and compared relative to fingerprint identification performance. The evaluation and comparison criteria using database of 80 persons with 12 fingerprint samples from each person. The multilayer perceptron neural network with back propagation training algorithm was used as a pattern classifier to evaluate the three mentioned image representation techniques. The obtained results showed that the average identification rate for the wavelet transform, Discrete Fourier transform and Gabor techniques are 97.9\%, 93.9\% and $88.1 \%$ respectively.

\section{INTRODUCTION}

Fingerprint identification is one of the most important biometric technologies since the unchangeable fingerprints during human life span and the uniqueness of each individual's fingerprints. A fingerprint is the pattern of ridges and valleys on the surface of a fingertip. The uniqueness of the fingerprint can be identified by the characteristics and relationships of bifurcations and endings in ridge or valley. In order to compare two fingerprints, a set of invariant and discriminating features are extracted from fingerprint image as shown in figure 1.

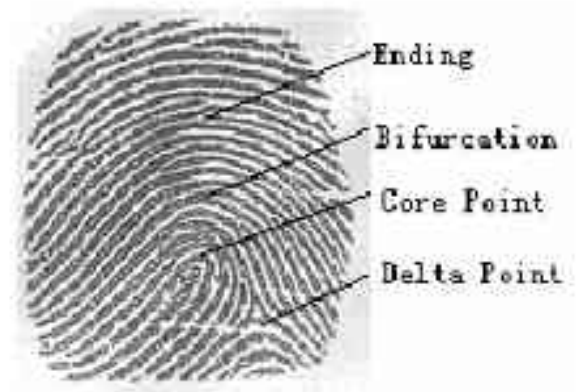

Figure 1: Examples of minutiae and singular points. 
As a global feature of fingerprints, the minutiae-based model is very important for automatic fingerprint recognition. Many algorithms have been proposed for the minutiae-based model, but their results are unsatisfactory, especially for poor quality fingerprint images [1]. In this paper, a model-based approach is used directly from grayscale image for image representation and feature extraction. In general the image recognition system design consists of four main stages: sensor, preprocessing, feature extraction and pattern recognition as shown in figure 2.

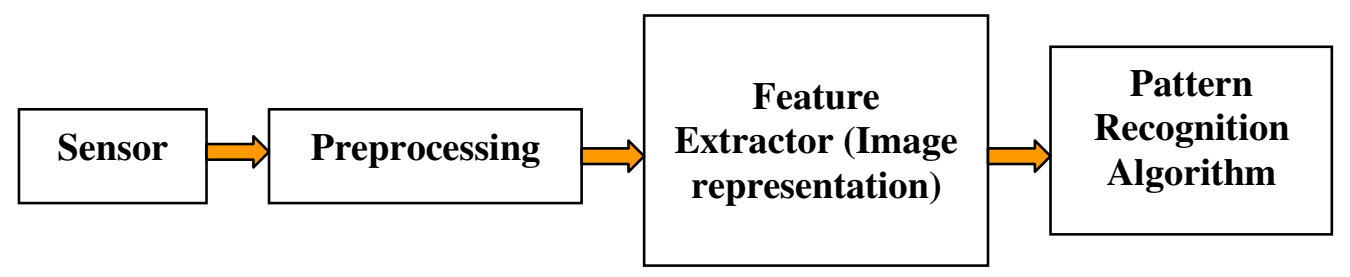

Figure 2: Simplified Fingerprint Recognition System.

\section{DATABASE}

The fingerprint database used in this study was acquired from 80 persons with 12 fingerprint samples from each person by using special optical scanner.

\section{PREPROCESSING}

In the preprocessing stage a histogram equalization used for fingerprint image enhancement to make the image clearer by increasing the contrast between ridges and furrows as shown in figure 3.

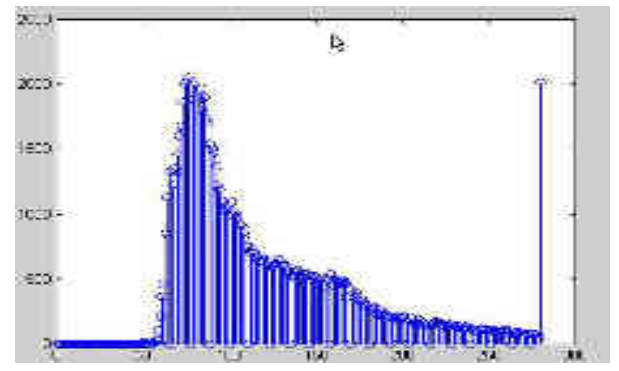

(a)

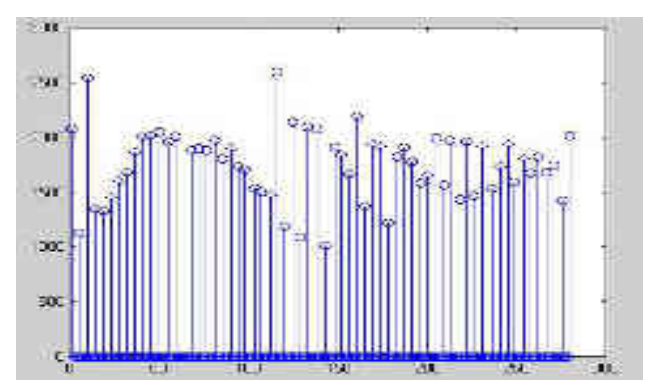

(b)

Figure 3: (a) Original histogram of a fingerprint image; (b) Histogram of a fingerprint image after the histogram equalization.

\section{FEATURE EXTRACTION}

The accuracy of the automatic fingerprint identification system depends, in the first place, on the image representation technique that is used for fingerprint images. The three different image representation techniques have been evaluated and compared 
relative to fingerprint identification. The three techniques are Gabor filters, Discrete Fourier transform and Wavelet transform. The multilayer perceptron neural network with back propagation training algorithm was used as a pattern classifier for evaluating the three mentioned image representation techniques. The following sections illustrate the implementation for each technique.

\subsection{Gabor Filters Algorithm}

The introduced fingerprint identification algorithm based on Gabor filters is illustrated in figure 4. The algorithm: (i) uses only one core point (ii) does not use estimated orientation field, and (iii) extracts information only in the central part of the fingerprint. A center point is detected in the input fingerprint images; a circular region around the center point is defined as the region of interest. The region of interest divided into concentric bands. Each band is divided into sectors. Each sector is normalized to constant mean and variance and filtered using a bank of Gabor filters to produce a set of filtered images. After we get the filtered images, we convert the image by 180 degree and get other filtered images again.

The variance of gray values in a sector is computed and taken as a feature vector. This will tell us the concentration of fingerprint ridges going in each direction in that part of the fingerprint. A feature vector, which we call finger-code, is the collection of the all features defined for each sector in each filtered image.

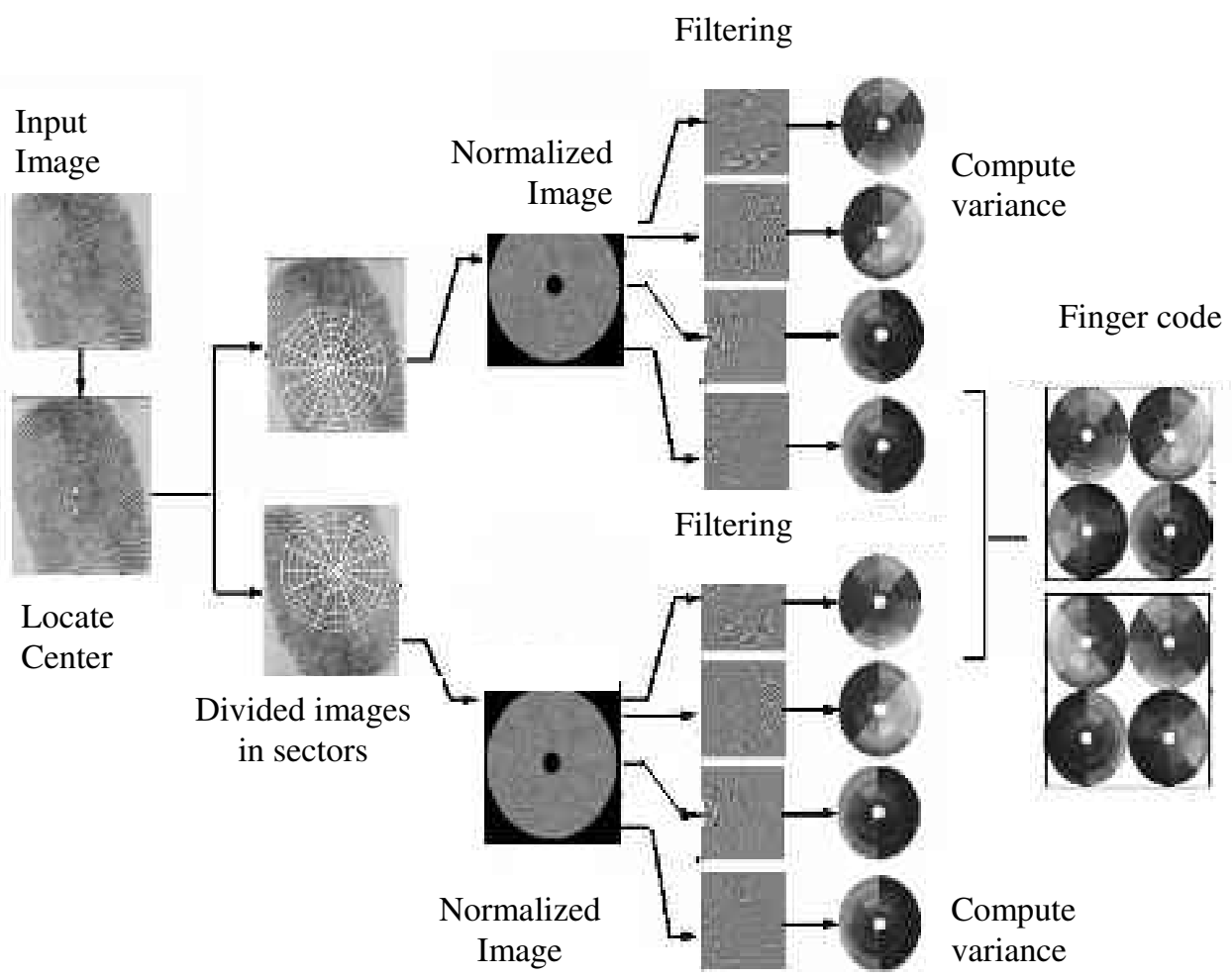

Figure 4: System design of the fingerprint identification algorithm using Gabor filters. 
The three main steps in implementing Gabor filters feature extraction algorithm are: (i) find a center point in the fingerprint image, (ii) filter the image in different directions using a bank of Gabor filters, and (iii) compute the variance of gray values in sectors around the center point of filtered images to define the feature vector or the fingercode.

\subsection{1 fingerprint center point determination}

The first step in the Gabor filters algorithm is to find the center point of a fingerprint image. Center point determination is done to find the point of most curvature by determining the normal of each fingerprint ridge, and then following those inwards towards the center. The following is the procedure we used [1].

- Apply a pixel-wise adaptive 2-D Gaussian lowpass filter to the fingerprint. The filter uses neighborhoods of size 5 pixels by 5 pixels to estimate the local gradient mean and standard deviation. This will help in reducing any noise that may cause spurious results in the following gradient calculations.

- Divide the input fingerprint image into non-overlapping blocks of size 10 pixels by 10 pixels.

- Determine the x and y magnitudes of the gradient at each pixel in each block, $G_{x}$ and $G_{y}$. This is done by taking the average of the two neighboring pixels.

- Apply the same 2-D Gaussian lowpass filter on the $\mathrm{x}$ and $\mathrm{y}$ gradients as above to smooth out the gradients.

- With each block, compute the slope perpendicular to the local orientation of each block using the following formula.

$$
\Theta=\frac{1}{2} \tan ^{-1}\left(\frac{\sum_{i=1}^{10} \sum_{j=1}^{10} 2 G_{x}(i, j) G_{j}(i, j)}{\sum_{i=1}^{10} \sum_{j=1}^{10}\left(G_{x}^{2}(i, j)-G_{y}^{2}(i, j)\right)}\right)+\frac{\pi}{2}
$$

- Only looking at blocks with slopes with values ranging from 0 to $\pi / 2$, trace a path down until you encounter a slope that is not ranging from 0 to $\pi / 2$ and mark that block.

- The block that has the highest number of marks will compute the slope in the negative $\mathrm{y}$ direction and output an $\mathrm{x}$ and $\mathrm{y}$ position which will be the center point of the fingerprint as shown in figure 5 .
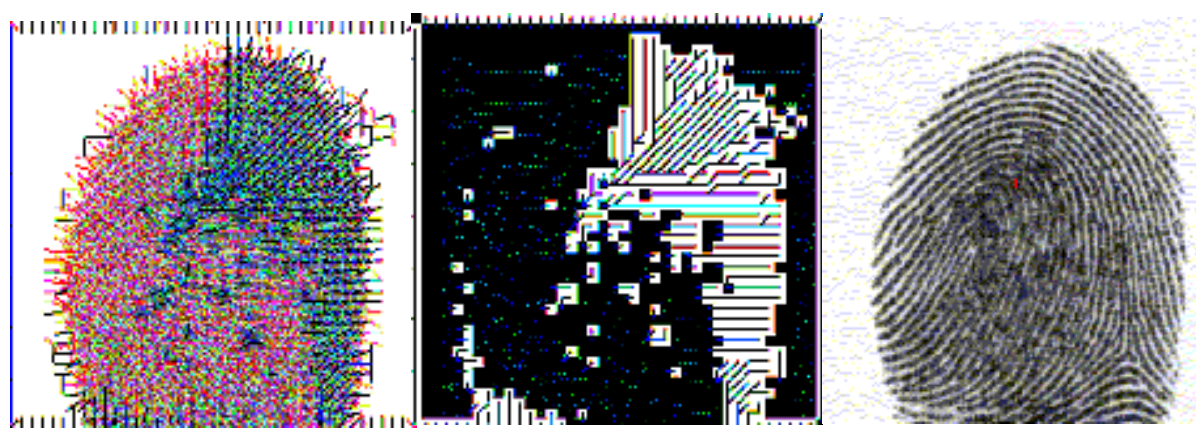

Figure 5: The center point determination process. 


\subsection{2 filtering of fingerprint image}

To remove noise and enhance the ridge and furrow structures, we filter the fingerprint image in different directions using a bank of Gabor filters [2, 3, 4]. Gabor filters are band-pass filters which have both orientation-selective and frequencyselective properties and have optimal joint resolution in both spatial and frequency domains [5]. Fingerprints have local parallel ridges and furrows, and well-defined local frequency and orientation. Properly tuned Gabor filters can remove noise, preserve the true ridge and furrow structures, and provide information contained in a particular direction in the image. An even symmetric Gabor filter has the following general form in the spatial domain:

$$
\begin{aligned}
G(x, y ; f, \theta) & =\exp \left\{\frac{-1}{2}\left[\frac{x^{2}}{\delta_{2}^{2}}+\frac{y^{2}}{\delta_{y}^{2}}\right]\right\} \cos \left(2 \pi f x^{\prime}\right), \\
x^{\prime} & =x \sin \theta+y \cos \theta, \\
y^{\prime} & =x \cos \theta-y \sin \theta,
\end{aligned}
$$

Where $f$ is the frequency of the sinusoidal plane wave along the direction $\theta$ from the $x$ axis, and $\delta_{x}$ and $\delta_{y}$ are the space constants of the Gaussian envelope along $x$ and $y$. In our experiments, we set the filter frequency $f$ to the average ridge frequency $(1 / K)$, where $K$ is the inter-ridge distance. Average inter-ridge distance is approximately 10 pixels in a 500 dpi fingerprint image.

After experimental implementation of the algorithm we select four different values for $\theta\left(0^{\circ}, 45^{\circ}, 90^{\circ}\right.$, and $\left.135^{\circ}\right)$ with respect to the $x$-axis. These four filters are shown in figure 6.

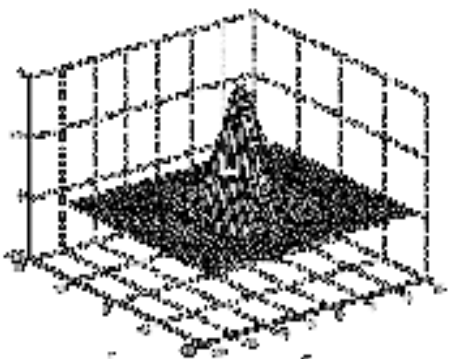

(a) 0 Orientation

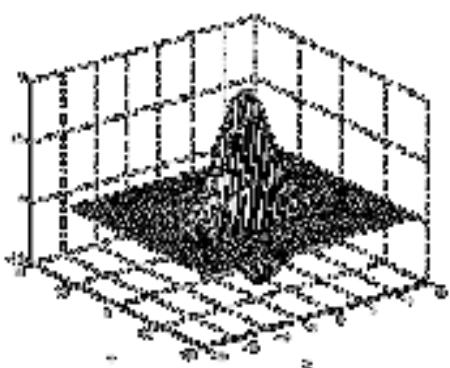

(c) 90 Orientation

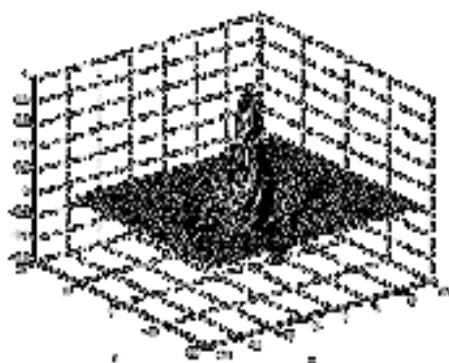

(b) 45 Orientation

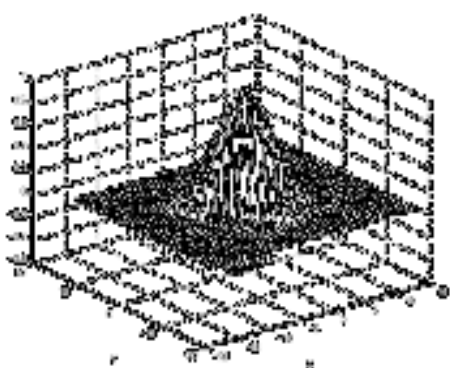

(d) 135 Orientation

Figure 6: Gabor filter $0, \pi / 4, \pi / 2$ and $3 \pi / 4$ orientation. 
The region of interest in a fingerprint image is convolved with each of these four filters to produce a set of four filtered images. Spatial domain convolving is rather slow, so multiplication in the frequency domain is done; however, this involves more memory to store real and imaginary coefficients. These four direction-sensitive filters capture most of the ridge directionality information present in a fingerprint. If the image is filtered in more than four directions using filters with broad bandwidth, there is a redundancy among the filters. If the image is filtered in less than four directions using filters with a narrow band width, the features can not tolerate image rotation and translation efficiently. At least four directions are required to capture the entire ridge information in a fingerprint. The values for $\delta x$ and $\delta y$ were empirically determined and set to 4.0 and 4.0 , respectively.

Before filtering the fingerprint image, we normalize the region of interest in each sector separately to a constant mean and variance. Normalization is done to remove the effects of sensor noise and finger pressure differences. Let $I(x, y)$ denote the gray value at pixel $(x, y), M i$ and $V i$, the estimated mean and variance of sector $\mathrm{Si}$, respectively, and $\mathrm{Ni}(x, y)$, the normalized gray-level value at pixel $(x, y)$. For all the pixels in sector $S i$, the normalized images are defined as:

$$
N_{1}(x, y)=\left\{\begin{array}{l}
M_{0}+\sqrt{\frac{V_{0} \times\left(I(x, y)-M_{i}^{2}\right)}{V_{2}}}, \text { if } I(x, y)>M_{i} \\
M_{0}-\sqrt{\frac{V_{0} \times\left(I(x, y)-M_{i}^{2}\right)}{V_{i}}}, \text { otherwise }
\end{array}\right.
$$

Where $\mathrm{M}_{0}$ and $\mathrm{V}_{0}$ are the desired mean and variance values respectively. Normalization is a pixel-wise operation which does not change the clarity of the ridge and furrow structures. If normalization is done on the entire image, then it can not compensate for the intensity variations in the different parts of the finger due to finger pressure differences. Normalization of each sector separately alleviates this problem. For our experiments, we set both $\mathrm{M}_{0}$ and $\mathrm{V}_{0}$ to a value of 100 .

\subsubsection{Feature vector}

Let $F_{i \theta}(x, y)$ be the $\theta$-direction filtered image for sector $S i$. For $\mathrm{V} i, i=0,1, \ldots$, 63 and $\theta €\left[0^{\circ}, 45^{\circ}, 90^{\circ}, 135^{\circ}\right]$, a feature is the variance $V i_{\theta}$, defined as:

$$
V_{i \theta}=\sqrt{\sum_{K_{i}}\left(F_{i \theta}(x, y)-P_{i \theta}\right)^{2}}
$$

Where $K i$ is the number of pixels in $S i$ and $P_{i \theta}$ is the mean of pixel values of $F_{i \theta}(x, y)$ in $\mathrm{Si}$. The variance of each sector in each of the four filtered images defines our feature vector. The resulting variance values (number of Gabor filters $\times$ number of sectors $\times 2$ rotated images) will be as the feature vector of the fingerprint scan. 


\subsection{4 gabor filters algorithm implementation}

The main factors were used in order to obtain highest identification performance of the Gabor filter algorithm are:

(i) The number of concentric bands.

(ii) The number of sectors per band.

(iii) The number of Gabor filters.

In our experiment we tried the values 3, 4 and 5 of bands and the values 12, 16 and 20 of sectors and the values 4,6 and 8 of bank of Gabor filters. The obtained experimental results are illustrated in table 1:

Table 1: Gabor filters algorithm experimental results.

\begin{tabular}{|c|c|}
\hline \# Bands & Id. rate \\
\hline 3 & $79.7 \%$ \\
\hline 4 & $88.125 \%$ \\
\hline 5 & $85.15 \%$ \\
\hline
\end{tabular}

\begin{tabular}{|c|c|}
\hline \# Sectors & Id. rate \\
\hline 12 & $85.9 \%$ \\
\hline 16 & $88.125 \%$ \\
\hline 20 & $77.5 \%$ \\
\hline
\end{tabular}

\begin{tabular}{|c|c|}
\hline $\begin{array}{c}\text { \# Gabor } \\
\text { filters }\end{array}$ & Id. rate \\
\hline 4 & $88.125 \%$ \\
\hline 6 & $86.7 \%$ \\
\hline 8 & $84.3 \%$ \\
\hline
\end{tabular}

The optimum values of concentric bands are 4, sectors per each band are 16 and number of gabor filters are 4.

N.B. the identification rate is the number of the correct identified images divided by the total images.

\subsection{Discrete Fourier Transform (DFT) Algorithm}

Utilizing the Discrete Fourier Transform filters to reduce the dimension dramatically and extract the discriminant features. The proposed method explores the capability of DFT and directional filtering in dealing with low quality images. Classification performance highly depends on the preprocessing steps where various ways to extract and represent distinguishable features among classes can be applied. The features generated after the preprocessing steps are fed into a neural network classifier. The DFT is a useful computational tool that provides an efficient means for detecting directionality or periodicity in the frequency domain and removing noise by deleting high frequency coefficients [6]. In the Discrete Fourier Transform (DFT), thresholding the high frequency coefficients corresponds to reducing the noise effects, while the low frequency coefficients provide a trigonometric interpolation via a finite linear combination of sines and cosines of the various frequencies [7].

\subsubsection{Feature extraction}

The following steps illustrate the DFT algorithm implementation as shown in figure 7:

i. Divide the input image into several blocks.

ii. Transform each 2-Dimensional block to 1-Dimensional vector.

iii. Apply 1-Dimensional Discrete Fourier Transform (DFT) at each vector.

iv. Get $\log$ of the magnitude of the DFT output.

v. Take several coefficients (Cepstral coefficients) to represent each block.

vi. The total number of coefficients will be a finger code. 


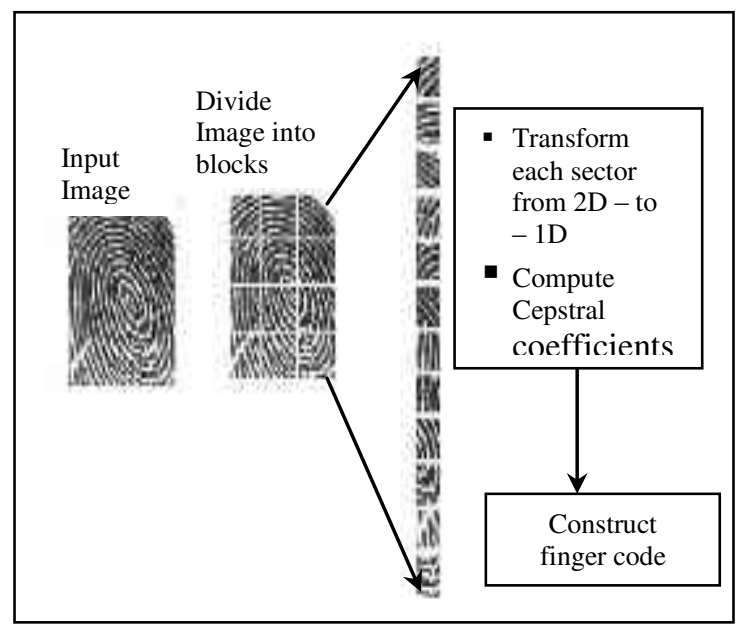

Figure 7: Discrete Fourier Transform for fingerprint representation.

\subsubsection{Discrete Fourier Transform Algorithm Implementation}

The main factors were used in order to obtain highest identification performance DFT algorithm are:

(i) The number of blocks.

(ii) The number of cepstral coefficients.

In our experiment we tried the values 12,16, 20 and 24 of cepstral coefficients and the values 1, 4, 6, 12, 16 and 24 of blocks. The obtained experimental results are illustrated in figures 8 and $\mathbf{9}$. The optimum values of blocks are 12 and number of cepstral coefficients are 16 .

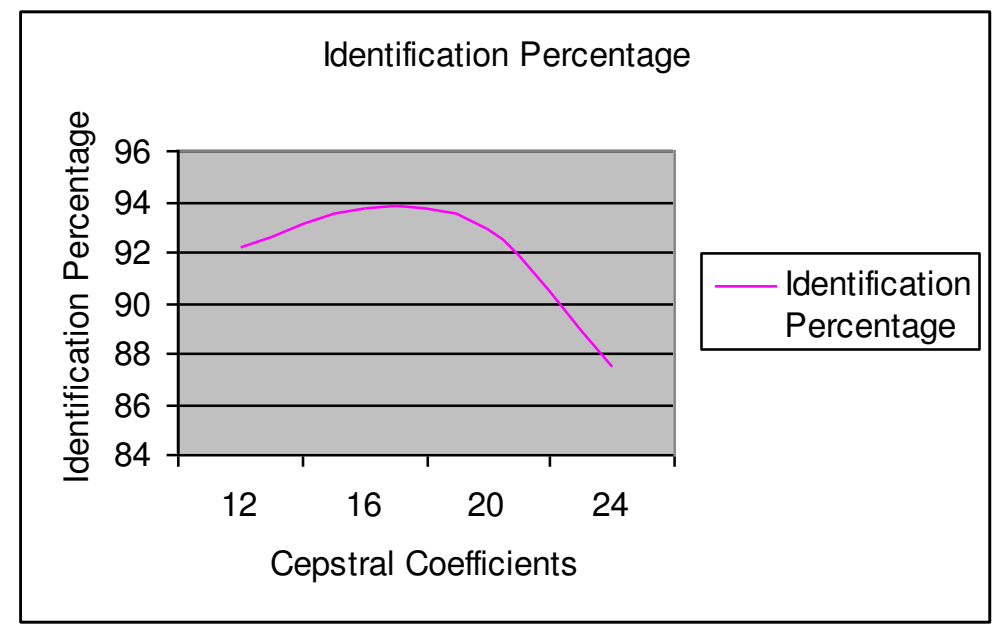

Figure 8: Cepstral coefficients optimum values experimental results. 


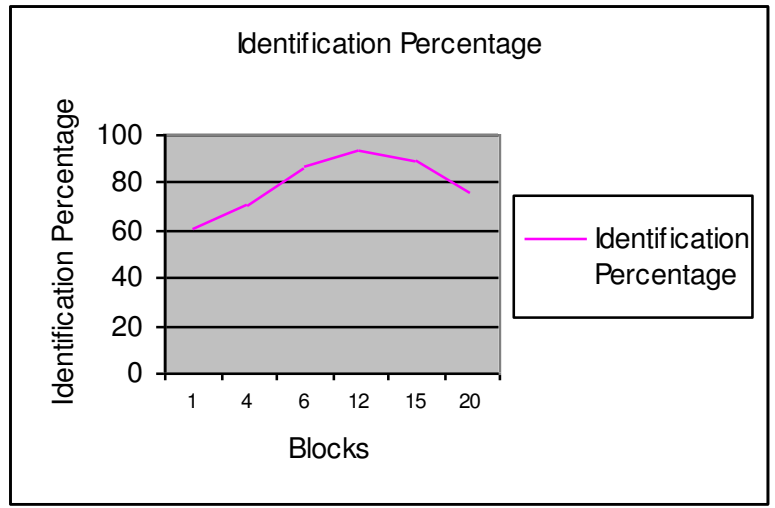

Figure 9: blocks optimum values experimental results.

\subsection{Wavelet Transform Algorithm (WT)}

In order to extract some information we have got some set processes available as we see before, Gabor filters, Discrete Fourier Transform (DFT) and Discrete Wavelet Transform (DWT). Each method has got some pros and cons in every domain, which restrict its use in that domain. DFT gives excellent performance in the frequency domain, but their performance in the time domain is restricted. The wavelet coefficients Cleary show the exact location the discontinuity in the time domain. Wavelet Transform is capable of revealing aspects of data such as breakdown points, trends, discontinuities in higher derivatives and self similarity that other signal analysis techniques won't be able to capture. Wavelet Transform can also compress or de-noise a signal without appreciable degradation [8].

\subsubsection{Discrete Wavelet Transform}

Dilations and translations of the "Mother function," or "analyzing wavelet" $\Phi(\mathrm{x})$; define an orthogonal basis, the wavelet basis [8]:

$$
\Phi_{(s, l)}(x)=2^{-\frac{\pi}{2}} \Phi\left(2^{-s} x-l\right)
$$

The variables " $s$ " and " $l$ " are integers that scale and dilate the mother function $\Phi$ to generate wavelets, such as a Daubechies wavelet family which we used in our algorithm. The scale index $s$ indicates the wavelet's width, and the location index $l$ gives its position. Notice that the mother functions are rescaled, or "dilated" by powers of two, and translated by integers.

The DWT is computed by successive lowpass and high pass filtering of the discrete time-domain signal. This is called the Mallat algorithm or Mallat-tree decomposition. Its significance is in the manner it connects the continuous time multi resolution to discrete-time filters as shown in figure 10. In figure 10, the signal is denoted by the sequence $x[n]$, where $n$ is an integer. The low pass filter is denoted by G0 while the high pass filter is denoted by H0. At each level, the high pass filter produces detail information, $\mathrm{d}[\mathrm{n}]$, while the low pass filter associated with scaling function produces coarse approximations, $\mathrm{a}[\mathrm{n}]$. 


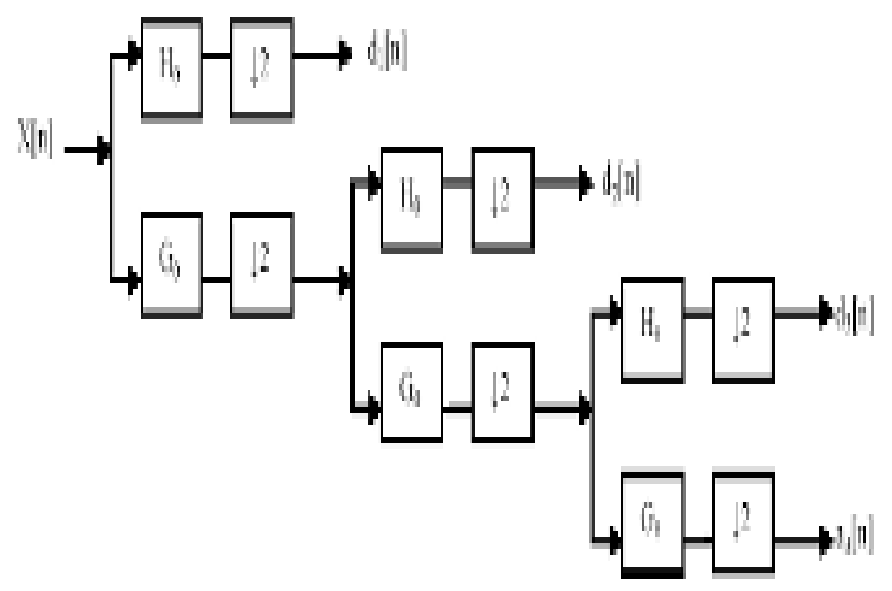

Figure 10: Three-level wavelet decomposition tree.

\subsubsection{Wavelet Transform Algorithm Implementation}

The following steps illustrate the Wavelet transform algorithm implementation:

i. Apply the two Daubechies decomposition wavelet filters to decompose the input image into four parts:
a. Low - Low part.
b. Low - High part.
c. High - Low part.
d. High - High part.

This will be considered as the first level of the Wavelet decomposition.

ii. Apply the Daubechies decomposition wavelet filters to decompose the "Low Low part" and get the output ( $2^{\text {nd }}$ level of Wavelet decomposition).

Repeat step iii several times till get the minimum accepted identification performance. In our experiment we tried the $1^{\text {st }}, 2^{\text {nd }}, 3^{\text {rd }}, 4^{\text {th }}$ and $5^{\text {th }}$ levels of wavelet decomposition to the fingerprint image. The obtained experimental results are illustrated in table 2:

Table 2: Wavelet algorithm experimental results.

\begin{tabular}{|c|c|}
\hline \# Wavelet Level & Identification Percentage \\
\hline 1 & 99 \\
\hline 2 & 99 \\
\hline 3 & 98.4 \\
\hline 4 & 88.2 \\
\hline 5 & 77.3 \\
\hline
\end{tabular}

Number of wavelet decomposition levels in our experimental results indicates that the 3 levels give good image representation results. 


\section{PATTERN RECOGNITION STAGE}

We used artificial neural network for the recognition stage with multilayer perceptron momentum and adaptive learning rate back-propagation training algorithm.

\section{COMPARISON AND EXPERIMENTAL RESULTS}

Our results are based on the following:

1- Get the fingercode array from each feature extraction technique that are introduced previously (Gabor filters, Discrete Fourier transform and wavelet transform).

2- Using 8 different patterns from each of the 80 person for training the Neural Network classifier.

3- Using 4 different patterns from each of the 80 person for testing the system identification capability.

\subsection{Experimental Results}

The results, obtained from Gabor filters, DFT and Wavelet algorithms after implementation, are as the follows:

\begin{tabular}{|l|c|c|c|}
\hline \multicolumn{1}{|c|}{ Comparison item } & Gabor Filters & DFT & Wavelet \\
\hline Input Image & $150 \times 200(30,000)$ & $\begin{array}{c}150 \times 200 \\
(30,000)\end{array}$ & $\begin{array}{c}150 \times 200 \\
(30,000)\end{array}$ \\
\hline $\begin{array}{l}\text { Calculated coefficients } \\
\text { (Input to classifier) }\end{array}$ & $\begin{array}{c}4 \text { Gabor filters x 16 Sector } \\
\text { x 4 Bands x 2 } \\
\text { '180 rotated image' (512) }\end{array}$ & $\begin{array}{c}16 \text { Cepstral } \\
\text { coefficients x 12 } \\
\text { blocks (192) }\end{array}$ & $\begin{array}{c}27 \times 21 \\
(567)\end{array}$ \\
\hline Data reduction ratio & $1: 58.59375$ & $1: 156.25$ & $1: 52.9$ \\
\hline $\begin{array}{l}\text { Training result } \\
\text { (80 person x 8 patterns) }\end{array}$ & $88.75 \%$ & $94.375 \%$ & $98.125 \%$ \\
\hline $\begin{array}{l}\text { Testing result } \\
\text { (80 person x 4 patterns) }\end{array}$ & $86.875 \%$ & $93.125 \%$ & $97.5 \%$ \\
\hline $\begin{array}{l}\text { Total } \\
\text { (including training) }\end{array}$ & $88.125 \%$ & $93.958 \%$ & $97.916 \%$ \\
\hline
\end{tabular}

Fingerprint identification algorithm based on Wavelet transform gives the highest identification performance as illustrated in figure 11.

\section{CONCLUSION}

Feature extraction based on the wavelet transform technique gives the highest fingerprint identification performance compared to Gabor filters and Discrete Fourier transform algorithms.

The Discrete Fourier transform algorithm gives us the highest data reduction ratio and this may direct us to use it in the applications required high compression ratio rather than identification performance. 


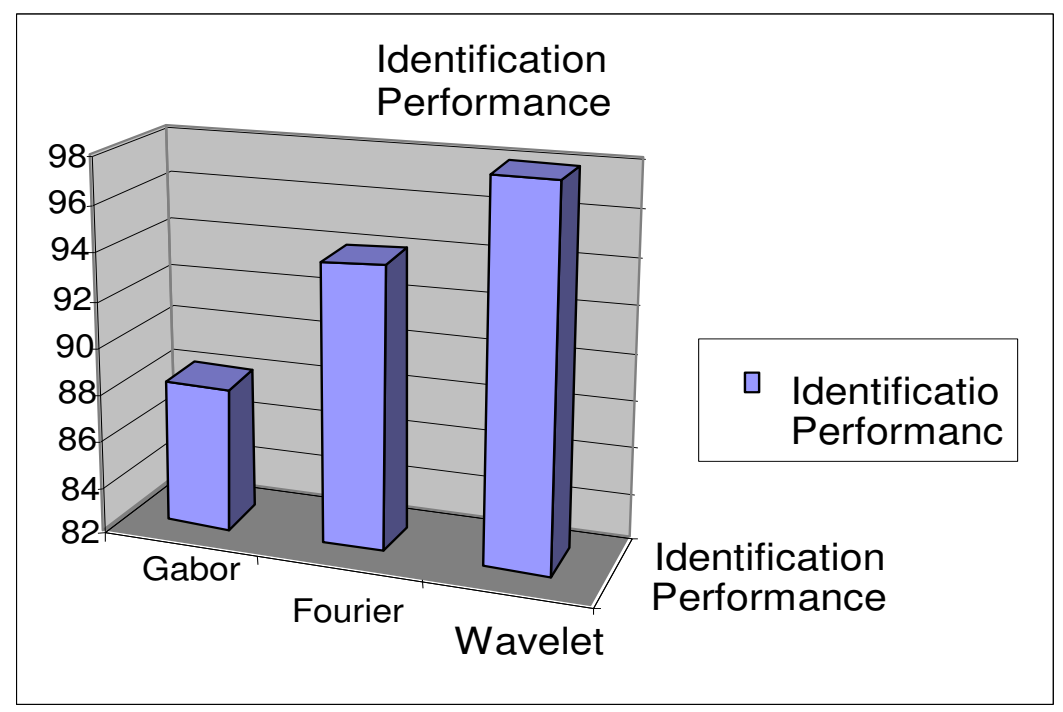

Figure 11: Identification results from three feature extraction techniques (Gabor filters, DFT and Wavelet transform).

\section{REFERENCES}

[1] P. Jonathon, Philips, Alvin Martin, C.L. Wilson, Mark, Przybocki, "An Introduction to Evaluating Biometric systems", IEEE Computer., Vol. 26, No. 7, pp. 56-63, February 2000.

[2] U.S. Department of Justice document SL000386, March 2000. Online: http://www.forensicvidence.comlsite/ID/IDfuValidation.html

[3] K. Jain, L. Hong, S. Pankanti, and Ruud Bolle, "An Identity Authentication System Using Fingerprints," Proceedings of the IEEE., Vol. 85, No.9, pp. 1365- 1388, 1997 .

[4] D. Maio and D. Maltoni, "Direct Gray-Scale Minutiae Detection in Fingerprints," IEEE Trans. Pattern Anal and Machine Intel., Vol. 19, No.1, pp. 27-40, 1997.

[5] K. Karu and A. K. Jain. "Fingerprint classification." Pattern Recognition, Vol. 29, No. 3, pp. 389-404, 1996.

[6] A. P. Fitz and R. J. Green, "Fingerprint classification using hexagonal fast Fourier transform," Pattern Recognition, Vol. 29, No. 10, pp. 1587-1597, 1996.

[7] A.V. Oppenheim, A.S. Willsky, and I.T. Young. "Signals and Systems". PrenticeHall, pp. 238-312, 1983.

[8] M. Vetterli and C. Herley, "Wavelets and Filter Banks: Theory and Design," IEEE Transactions on Signal Processing, Vol. 40, No. 6, pp. 2207-2232, 1992. 


\section{نظام التعرف الآلي على البصمات}

\section{ما نهى محمد أحمد صالح}

يقوم هذا البحث بتقييم ثناث طرق مختلفة من طرق تمثيل الصور لمعرفة مدى دقة كل

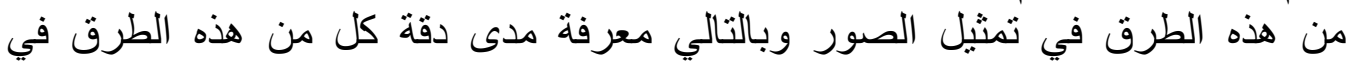

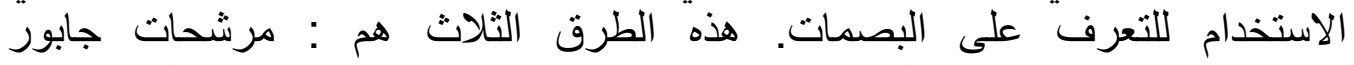
(Fourier Transform) ، محوف (Gabor Filters) (Wavelet Transform)

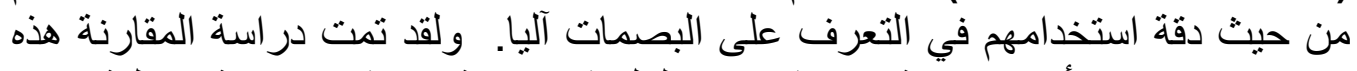

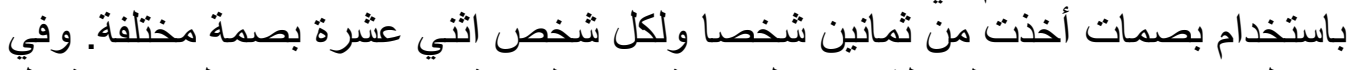

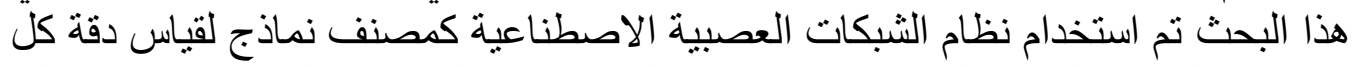

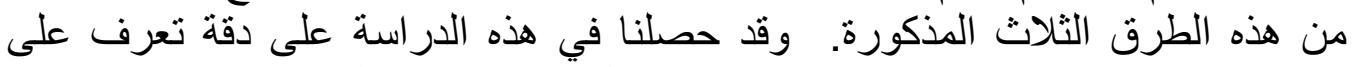

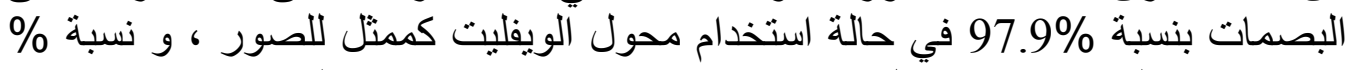
93.9 في حالة استخدام محول فورير ، و و نسبة \% 88.1 في حالة استخدام مرشحات 